\title{
Kernos
}

Revue internationale et pluridisciplinaire de religion grecque antique

15 | 2002

Varia

\section{Aiatos et Polycléia. Du mythe à l'histoire}

\section{Babis G. Intzésiloglou}

Édition électronique
URL : http://journals.openedition.org/kernos/1386

DOI : 10.4000/kernos. 1386

ISSN : 2034-7871

\section{Éditeur}

Centre international d'étude de la religion grecque antique

Édition imprimée

Date de publication : 1 janvier 2002

ISSN : 0776-3824

\section{Référence électronique}

Babis G. Intzésiloglou, «Aiatos et Polycléia. Du mythe à l'histoire ", Kernos [En ligne], 15| 2002, mis en ligne le 21 avril 2011, consulté le 01 mai 2019. URL : http://journals.openedition.org/kernos/1386 ; DOI : 10.4000/kernos. 1386 


\section{Aiatos et Polycléia. Du mythe à l'histoire}

Le mythe d'Aiatos et Polycléia a été conservé dans les Strategemata de Polyen ${ }^{1}$ et concerne l'arrivée des peuplades préthessaliennes venues d'Épire dans la région de la future Thessalie, à l'est de l'Achéloos.

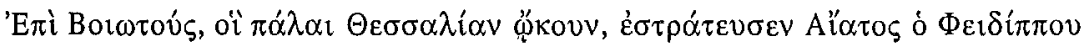

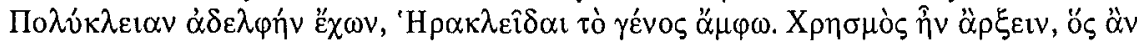

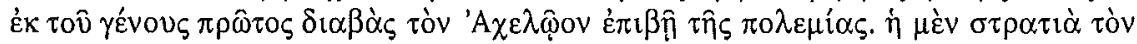

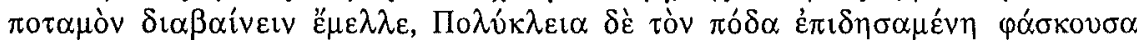

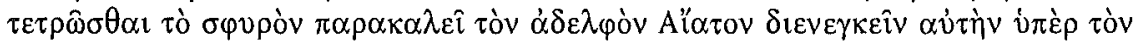

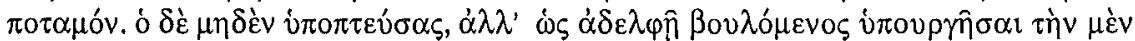

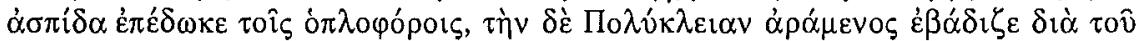

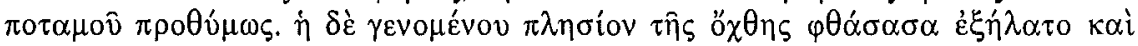

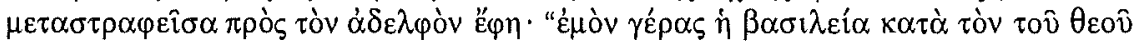

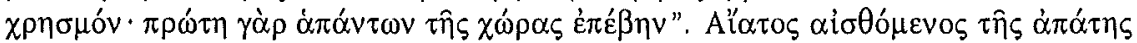

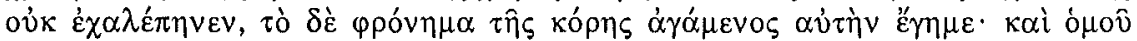

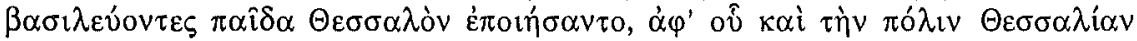
$\pi \rho 0 \sigma \eta \gamma o ́ p \varepsilon v \sigma \alpha v$.

Aiatos, fils de Pheidippos, ainsi que sa sœur Polycléia, tous deux issus de la race d'Héraclès, entreprirent une expédition contre les Béotiens. Il y avait eu un oracle selon lequel le premier de leur race à poser le pied en terre ennemie sur l'autre rive de l'Achéloos y deviendrait roi. Quand leur armée était prête à passer le fleuve, Polycléia, après avoir bandé son pied sous prétexte qu'elle s'était blessée à la cheville, demanda à son frère Aiatos de la faire passer de l'autre côté. Celui-ci, sans l'ombre d'un soupçon et désireux de lui porter secours, après avoir donné son bouclier aux soldats, prit de bon gré Polycléia dans ses bras et s'avança dans le fleuve. Mais elle, au moment où Aiatos atteignait l'autre rive du fleuve, sauta de ses bras et se retournant vers son frère lui dit : «Il m'appartient de régner sur cette terre en vertu de l'oracle du dieu, puisque c'est moi qui la première de tous ai posé le pied sur la terre ennemie ". Aiatos comprit la duperie dont il avait été l'objet, mais n'en fut point contrarié. Au contraire, par admiration pour la malice de la jeune fille, il l'épousa et ils devinrent tous deux rois de la région. Ils eurent un fils, Thessalos, d'après lequel on appela la région Thessalie.

1 VIII, 44. 


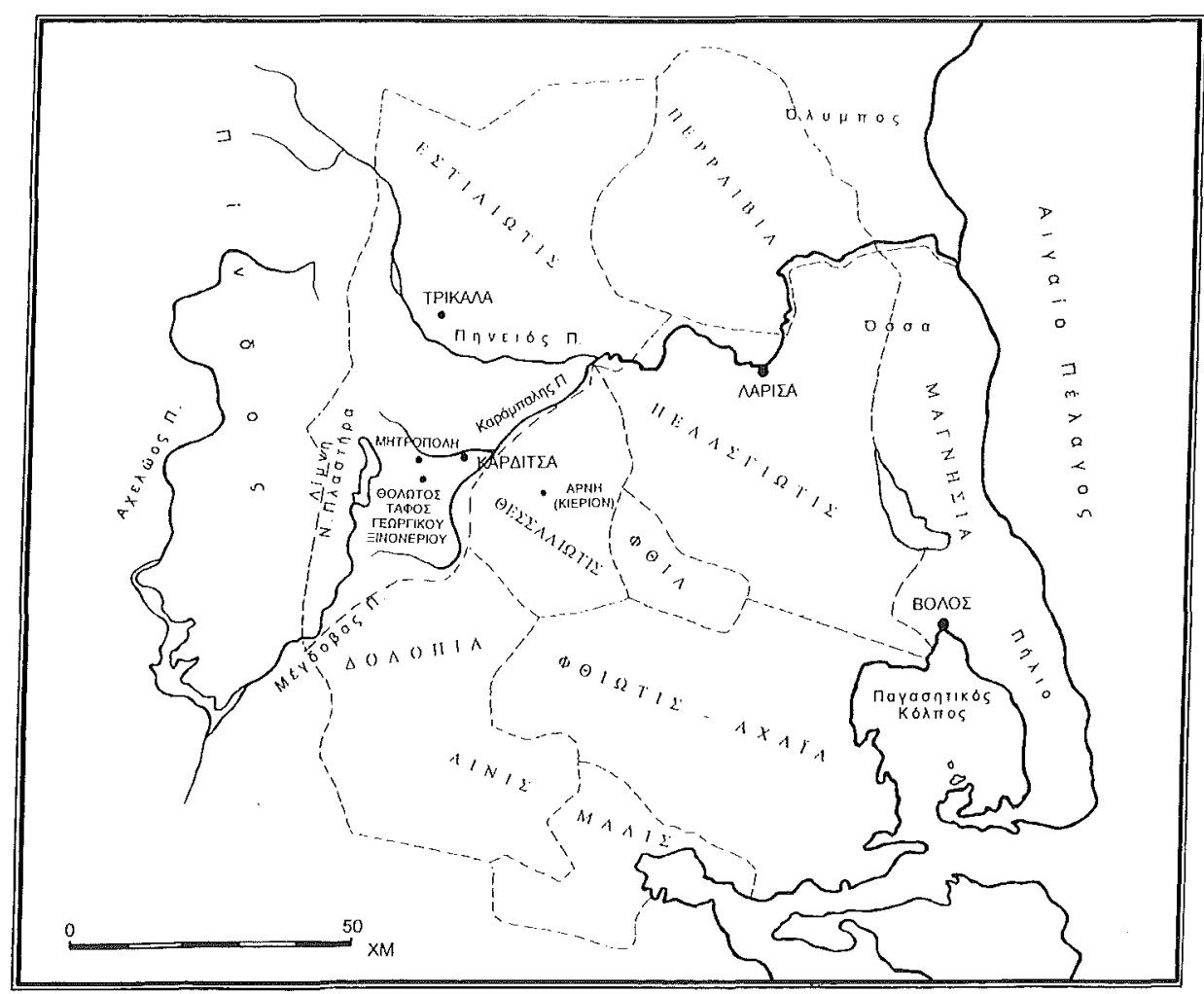

Il est évident qu'il s'agit d'un mythe fondateur, dont l'intrigue est simple et compréhensible sans explication. Il est en revanche particulièrement intéressant de pouvoir définir l'espace où le mythe prend place en utilisant les indications géographiques dont il fait mention, mais aussi d'autres renseignements fournis par la littérature grecque ancienne én les recoupant avec les découvertes archéologiques. Nous nous étions précédemment occupé de cette question ${ }^{2}$ et nous étions parvenu à certaines conclusions que nous allons reprendre ici de façon plus analytique.

On peut utiliser deux renseignements pour déterminer géographiquement la région dont le mythe fait mention : a) l'Achéloos est désigné comme limite de la région où habitaient le frère et la sœur, Aiatos et Polycléia, avant leur expédition militaire; b) les Béotiens sont clairement désignés dans le mythe comme les habitants de l'autre rive du fleuve, à l'époque, naturellement, où

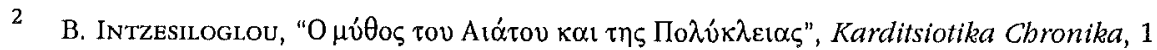
(1995), p. 11-18. 
ils étaient encore installés dans la région de ce qui sera appelé par la suite Thessalie ( $c f$. la carte ci-contre).

En ce qui concerne l'Achéloos, les témoignages des textes anciens donnent deux fleuves portant ce nom dans la très vaste région de Thessalie. Le premier correspond au fleuve actuel du même nom qui prend sa source dans la masse montagneuse du Pinde ${ }^{3}$. Le second fleuve portant le nom d'Achéloos (non identifié à ce jour) est mentionné dans la région de Lamia et les habitants des villes qui ont été fondées le long de ce fleuve sont mentionnés sous le nom de Parachéloïtes ${ }^{4}$. Sur la base de ces données, la recherche actuelle attribue également le nom d'Achéloos à l'un de ses principaux affluents, l'actuel Megdovas, et situe les Parachéloïtes dans la région du plateau de Névropolis et de l'actuel lac N. Plastiras.

En ce qui concerne la présence des Béotiens dans le mythe, on sait que les Béotiens étaient installés dans la région de la future Thessalie avec pour siège Arnè, que l'on identifie comme la ville devenue ensuite l'antique Kierion $^{6}$ ( $c f$. la carte ci-contre).

Le rapprochement entre les deux indications d'ordre géographique mentionnées par le mythe montre clairement que la région occupée par les Béotiens était bornée à l'ouest par l'Achéloos et s'étendait à l'est au moins jusqu'à la ville d'Arnè, dans la plaine, qui était leur siège. Le fait que le frère et la sœur, les héros du mythe, se soient emparés d'une partie de la superficie du territoire béotien, et non de la totalité, est également avéré par le fait que les Béotiens ont plus tard été définitivement éloignés d'Arnè par Thessalos, le fils d'Aiatos et Polycléia, et ont été contraints d'émigrer dans la région de la future Béotie ${ }^{7}$.

Selon les données connues jusqu'à ces dernières années, il n'y avait, dans la région située entre Arnè et l'Achéloos, que des indices du lien entre le mythe et une région précise plus restreinte. Tous ces indices sont concentrés autour de la ville et dans le territoire de l'antique Métropolis ${ }^{8}$ :

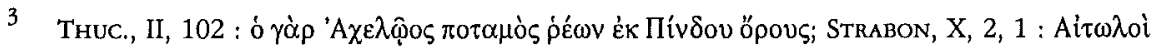

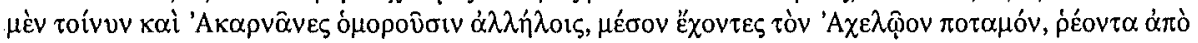

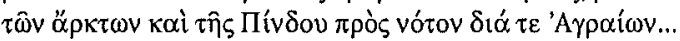

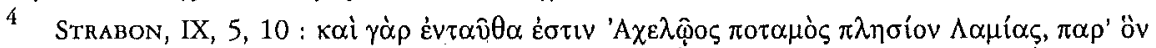

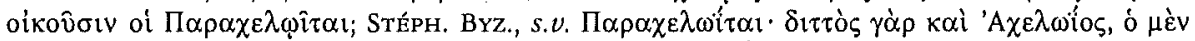

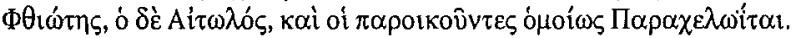

5 Fr. STäHLIN, Das bellenische Thessalien, Amsterdam, $1967^{2}$, p. 146, n. 17 et 18.

6 Ibid., p. 85 et $130-131$.

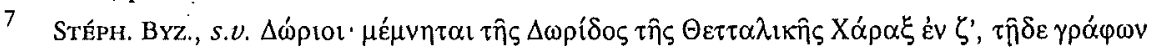

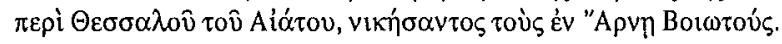

8 L'antique Métropolis se trouve à $9 \mathrm{~km}$ à l'ouest de Karditsa à l'emplacement de l'actuel village du même nom (autrefois "Palaiokastro ") et appartient à l'antique tétrade de l'Hestiaiotide. D'après Strabon (IX, 5, 17), l'ancienne cité fut créée à la suite du synécisme de trois obscurs villages des environs. On trouve la plus ancienne attestation de Métropolis dans une inscription de Delphes qui date de 358 av. J.-C. Cette date est le 
a) l'existence, sur le site de Gheorghikon-Xinonérion, de la seule grande tombe mycénienne à tholos de Thessalie occidentale et la mieux conservée de toute la Thessalie, qui constitue une preuve sérieuse de l'existence dans la région d'une installation humaine organisée au moins depuis le xiv siècle avant $\mathrm{J} .-\mathrm{C} .{ }^{9}$;

b) le motif, au revers d'une pièce de monnaie en bronze de Métropolis, d'une protomé de taureau à visage humain, habituellement interprété comme la représentation d'une divinité fluviale ${ }^{10}$. Dernièrement, on a soutenu que le motif de la pièce de monnaie trouvée à Métropolis pourrait être interprété comme la représentation du fleuve Achéloos divinisé ${ }^{11}$, dont le culte, en dehors du fait qu'il était très largement répandu dans le monde grec antique, pourrait également être dû au fait que le fleuve constituait vraisemblablement la limite occidentale du territoire de l'antique cité, que le nom d'Achéloos ait été porté seulement par le fleuve ou par son affluent le Megdovas ${ }^{12}$;

c) le nom même de Métropolis, qui renvoie à la création d'un centre urbain construit sur la base d'une origine ethnique commune aux habitants de la ville.

La fouille de la tombe à tholos de Gheorghikon-Xinonerion ${ }^{13}$ a apporté, au cours des dernières années, de nouveaux éléments qui confortent les indices déjà dégagés et lient désormais l'emplacement du mythe à la région de Métropolis.

La tombe à tholos se trouve à environ un kilomètre au sud de la ville antique, aux confins de sa nécropole qui s'étend dans cette direction ( $c f$. carte). Pour construire la tombe, on a taillé la roche tendre d'une colline isolée au lieu-dit «Koufia Rakhi », qui constitue la dernière colline avant la plaine, dans l'un des contreforts inférieurs du massif montagneux principal de la chaîne du Pinde. Au sommet de la colline se trouvait une tombe que l'on discerne à peine aujourd'hui.

terminus ante quem de la fondation de la cité, alors que les découvertes des fouilles menées jusqu'à ce jour ne permettraient pas de dater sa fondation au $\mathrm{v}^{\mathbf{e}}$ siècle av. J.-C.

9 BCH 44 (1920), p. 395; AD 16 (1960) Chron., p. 171; O. PeLON, Tholoi, tumuli et cercles funéraires, Paris, 1976, p. 252-253; W.G. Cavanagh, R.R. Laxton, "The Structural Mechanics of the Mycenaean Tholos Tomb", ABSA 76 (1981), p. 109-140.

10 E. Rogers, The Copper Coinage of Thessaly, London, 1932, p. 134, $\mathrm{n}^{\circ} 410-411$.

11 H.P. Isler, Acheloos, Bern, 1970, p. 89. A. Moustaka (Kulte und Mythen auf thessalischen Münzen, Würtzburg, 1983, p. 53) interprète le motif de la monnaie comme la représentation d'un dieu fluvial de la région.

12 Nous écartons l'identification de l'Achéloos du mythe d'Aiatos avec le fleuve de Phtiotide mentionné par Strabon, qui se trouve à côté de Lamia (cf. note 4), parce que, hormis le fait que la localisation de ce fleuve n'a pas encore été déterminée, cela serait largement en contradiction avec d'autres renseignements fournis par la littérature antique qui mentionnent l'origine épirote des peuplades préthessaliennes qui vinrent dans la région ultérieurement appelée Thessalie.

$13 A D 52$ (1997) Chron. (sous presse). 
La tombe à tholos, connue depuis 1917, a conservé sa tholos entière (hauteur et diamètre de la tholos : $8,80 \mathrm{~m}$ ), son ouverture, cachée par d'énormes linteaux (10,30 $\mathrm{m}$ de long), et son dromos (10,60 $\mathrm{m}$ de long) fermé par un mur à son extrémité extérieure. La chambre circulaire est bâtie en pierres calcaires de taille relativement petite et a été à maintes reprises l'objet de trafic d'antiquités. Le sol de la chambre, constitué par la roche d'origine, a été détruit par le creusement de grands trous et de plus petits, et, dans les rares endroits où il a été sauvegardé, on a constaté l'existence d'un étroit conduit creusé dans la roche, qui part du milieu de la paroi opposée à l'entrée et, sủivant la ligne circulaire des parois du côté ouest de la chambre, arrive à l'ouverture d'où, en ligne droite et presque caché par de petites dalles de calcaire, il coupe par le milieu l'ouverture et le dromos, et aboutit à l'extérieur de la tombe, à quelques mètres au sud du mur qui ferme le dromos.

Lors du dégagement du sol de la tholos et de l'ouverture, on a trouvé de rares morceaux de poterie sans décor qu'il est difficile de dater, quelques intailles, un petit peigne en os, un scarabée et une bague en or avec un motif sur son chaton. Enfin, à l'extérieur du mur qui ferme le dromos, on a trouvé une coupe à une seule anse qui pourrait dater de la période HR III B (c'est-àdire du XIII ${ }^{\mathrm{e}}$ siècle avant $\mathrm{J}$-C.).

Ce qui intéresse le mythe d'Aiatos et Polycléia réside principalement dans les découvertes de la fouille effectuée à l'extérieur du dromos de la tombe. $\mathrm{Au}$ sud du dromos et sur une superficie d'environ $530 \mathrm{~m}^{2}$, on a trouvé des tas de pierres composés de pavés calcaires et surtout de galets de rivière sur lesquels on avait placé des centaines d'ex-voto du type des petites figurines de cavaliers en terre cuite modelées à la main et des couteaux en fer de tailles et de formes très diverses.

L'ensemble des découvertes mentionnées ci-dessus, parallèlement à la présence de deux socles sans inscription destinés à des colonnes en forme de pilastres et d'un endroit qui conserve des traces de feu bien marquées avec des fragments d'os d'animaux calcinés, montre que, de toute évidence, le lieu fonctionnait comme sanctuaire d'un culte des ancêtres comparable à d'autres sanctuaires découverts en Attique ou dans le Péloponnèse près de tombes à tholos mycéniennes.

La découverte d'un lieu de culte des ancêtres lié à une tombe à tholos mycénienne, localisé pour la première fois en Thessalie, fait géographiquement remonter la zone attestée de ce type de sanctuaires vers le nord, jusqu'en Thessalie, et répond ainsi positivement à la question posée par la bibliographie à propos de l'absence jusqu'ici avérée de semblables sanctuaires en territoire thessalien.

Cependant, le rapprochement entre le sanctuaire et le mythe d'Aiatos et Polycléia a été opéré par la découverte au milieu de la zone sacrée en question d'une tuile en terre cuite de type laconien sur la surface convexe de laquelle est conservé un fragment d'inscription gravée datant du viI ${ }^{e}$ ou du vi 
siècle avant J.-C. où nous pouvons lire vers la gauche la mention Aiatiio (génitif singulier) ou Aiation (accusatif singulier) (Fig. 1), c'est-à-dire l'attestation qu'Aiatos est le propriétaire du sanctuaire ${ }^{14}$.

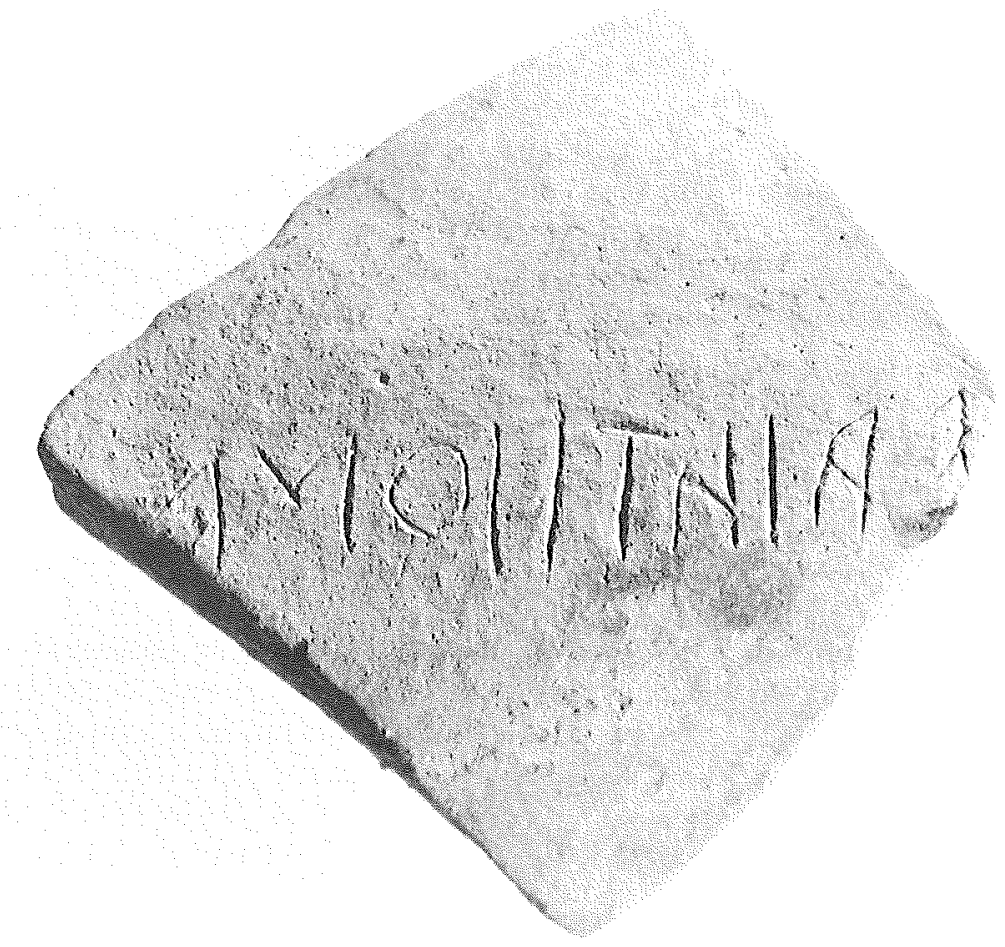

Le sanctuaire de la tombe à tholos de Gheorghikon-Xinonérion acquiert une identité grâce à la découverte de cette inscription et nous suggère parallèlement que le mythe d'Aiatos est antérieur $\mathrm{au}^{\prime} \mathrm{vi}^{\mathrm{e}}$ siècle avant J.-C. Il montre également que les habitants de la région n'adoraient pas un héros

14 Dimensions du morceau de tuile : L. $0,14 \times 1.0,132 \times$ h. 0,02 m. La hauteur des lettres oscille entre le $0,013 \mathrm{~m}$ pour l'omicron jusqu'à $0,02 \mathrm{~m}$ pour le iota. Le texte conservé de l'inscription incisée de droite à gauche est le suivant : ]EAIATIIONFi. Nous accordons la préférence à la lecture qui fait le lien avec le sanctuaire d'Aiatos, dans la mesure où l'interprétation des lettres TII (au milieu de l'inscription conservée) nous semble plus difficile si l'on reconnaît dans les quatre premières lettres (JEAIA) la terminaison d'un adjectif patronymique de genre féminin et dans les trois dernières lettres (ONF) le début du verbe $\delta \nu \epsilon \theta \epsilon \iota \kappa \epsilon$. Des appellations parallèles pour des sanctuaires sont attestées depuis l'antiquité comme, par exemple, le Théseion et l'Érechtheion à Athènes, le Pélopion (temenos) à Olympie, etc. - Je remercie mes collègues Mme Argyroula Doulgeri-Intzesiloglou pour la lecture de l'inscription et M. Angelos Matthaiou pour la discussion du problème. 
anonyme, comme on le croyait jusqu'à maintenant pour tous les sanctuaires qui ont été trouvés près de tombes à tholos mycéniennes ${ }^{15}$, mais le fondateur de la région qui était aussi le père de Thessalos et l'ancêtre de tous les Thessaliens. Il met aussi en lumière le fait que les habitants de la région, au $\mathrm{vII}^{\mathrm{e}}-\mathrm{vI}^{\mathrm{e}}$ siècle avant J.-C., étaient convaincus qu'Aiatos et. Polycléia avaient marché vers la plaine de Thessalie en s'emparant d'abord de leurs territoires, une fois passé l'Achéloos. Cela montre que le choix de la protomé de taureau à visage humain au revers de la pièce de monnaie en bronze n'était pas dû au hasard, mais qu'il pourrait être rapproché du mythe du premier fondateur de la région. Il montre aussi que la fondation de Métropolis et le rétablissement de son nom exact ne peuvent être étrangers à la conviction des habitants antérieurs d'avoir une origine commune due au fondateur de la région, l'Héraclide Aiatos ainsi qu'à sa sœur Polycléia.

Une tuile portant une inscription est ainsi devenue le fil qui conduit de l'horizon des mythes à celui de l'histoire.

Musée Archéologique de Volos

Babis G. INTZÉSILOGLOU

Athanasaki 1

GR - Volos

15 Carla Antonaccio, "Placing the Past: the Bronze Age in the Cultic Topography of Early Greece", in S.E. Alcock, R. OsBorne (éds), Placing the Gods, Oxford, 1994, p. 79-104. 